\title{
An asymptotic solution for transpired incompressible turbulent boundary layers
}

\author{
ATILA P. SILVA-FREIRE \\ Engineering Department, Cambridge University, Trumpington Street, Cambridge CB2 IPZ, U.K.
}

(Received 1 May 1987 and in final form 30 September 1987)

\begin{abstract}
In this work perturbation techniques are used to study the equations of mean motion for transpired incompressible turbulent boundary layers. One of the features of this approach is the use of multiple scales. Indeed, the solution of this problem requires the use of three distinct scales associated with the three flow regions: the inviscid region, the defect layer and the wall layer. In the construction of the wall and defect solutions, a new law of the wake is determined by comparison of these two solutions in an interval of common domain of validity. The matching conditions also yield a skin-friction equation for transpired turbulent boundary layers. The velocity profiles obtained from the law of the wake expression, and the values of the skin-friction coefficient predicted by the skin-friction equation for flows with both injection and suction of fluid, are compared with experimental data showing good agreement.
\end{abstract}

\section{INTRODUCTION}

THERE is considerable interest in the properties of turbulent boundary layers flowing over porous surfaces with injection or suction of flow through the surface. Indeed, several works have been presented in the literature which analyse both the theoretical and the experimental aspects of the problem of transpired turbulent boundary layers. Much of the theoretical studies on this topic have concentrated on obtaining expressions for the law of the wall and the law of the wake. These laws have normally been derived using the mixing-length theory of Prandtl together with the assumption that there is a region near the wall in which only inertia and turbulent terms are important in the $x$-momentum equation. This procedure, however, poses the difficulty of not providing proper boundary conditions to evaluate the parameters in the resulting expressions. Such parameters must then be determined with the aid of supplementary experimental data. Unfortunately, the general lack of agreement between the various sets of data makes such determination very difficult to accomplish. This is particularly true if one considers that those parameters depend critically on the assumed value of the skinfriction coefficient, and that most of the skin-friction values presented in the literature have been obtained with the use of some form of the momentum-integral equation, which tends to be very inaccurate.

The aim of the present work is to apply perturbation methods to the problem of transpired turbulent boundary layers, and show how bilogarithmic laws can be obtained for the wall and defect layers through this method. An important result of this approach is the derivation of a skin-friction equation for transpired turbulent boundary layers. The great advantage of the skin-friction equation over the momentumintegral equation for predictions of skin-friction coefficients, is that it is much less sensitive to small changes in injection velocity and in Reynolds number, and so it gives much more reliable results. To the author's knowledge this is the first derivation of such an equation. The additional relationship provided by this equation is here used to determine the various parameters in the law of the wake. The results are then cross checked comparing the defect layer solution with experimental data. The present formulation is shown to provide good predictions for flows with both injection and suction of fluid.

Singular perturbation techniques are required here since our problem is characterized by the necessity of working with multiple scales. The reason for this is that, as we shail see, turbulent and viscous forces are significant only in well-defined regions of the flow, the defect and the wall layers. The disturbances caused on the main flow by the blown or sucked fluid are on the other hand significant throughout the defect and wall layers and hence are accounted for by regular perturbation methods.

The procedure which leads to the derivation of the small singular parameters, $\varepsilon$ and $\hat{\varepsilon}$, is standard in turbulent boundary layer theory $[1,2]$ and we anticipate that for the transpired case these parameters are the same as for the unblown case. Hence the defect layer is scaled by $\delta=\varepsilon l$, where $\varepsilon\left(=u_{\tau} / u_{\infty}=\sqrt{ } \tau / \rho u_{\infty}^{2}\right)$ is the non-dimensional friction velocity and $l$ the inviscid length scale, whereas the wall layer is scaled by $\delta=\delta \hat{\varepsilon}=v / u_{\xi}$. The boundary conditions at the wall define the small regular parameter, $\bar{\varepsilon}=v_{\mathrm{w}} / u_{\infty}$.

In solving our problem we approximate the solutions in the two inner layers by suitable asymptotic expansions and use a rational approach to construct sets of governing equations for each of these layers. Afzal [3] studied this problem, however, he did not treat $\varepsilon$ and $\tilde{\varepsilon}$ as independent parameters and hence he obtained an incomplete set of equations and solutions. 


\section{NOMENCLATURE}

$A, B, C, D$ parameters in the law of the wall and law of the wake

a parameter in Stevenson's law of the wall

$C_{\mathrm{f}} \quad$ skin-friction coefficient

$d$ parameter in unblown law of the wall

$K \quad$ Von Karman's constant

$l \quad$ characteristic reference length

$P \quad$ pressure

$R \quad$ Reynolds number

$T_{x y}, T_{x x}, T_{y y} \quad$ Reynolds stress tensor components

$u_{\tau} \quad$ friction velocity

$U, V$ mean velocity components

$u^{\prime}, v^{\prime}$ velocity fluctuations

$X$ parameter in Stevenson's law of the wake

$y, \hat{y}$ normal coordinates in defect and inner regions

$w(y)$ Cole's wake function.

Greek symbols

$\delta \quad$ boundary layer thickness $\delta \quad$ wall layer thickness

$\varepsilon$ dimensionless friction velocity

$\tilde{\varepsilon} \quad$ dimensionless injection velocity

$\hat{\varepsilon} \quad$ wall layer small parameter

$\mu, v \quad$ viscosity, kinematic viscosity

$\theta \quad$ momentum thickness

$\pi \quad$ Cole's profile parameter

$\tilde{\pi} \quad$ profile parameter of wake component due to transpiration

$\rho$ density

$\tau$ Reynolds stress tensor components in defect region

$\psi \quad$ universal function in Stevenson's law of the wake.

Superscript

wall layer quantity.

Subscripts

$\infty$ external flow conditions

w conditions at the wall.
In particular, he was unable to derive expressions for the law of the wall and law of the wake similar to those obtained by Simpson [4], Stevenson [5, 6] and McQuaid [7]. The solutions obtained for adjacent layers in the present study are then shown to match in overlap domains so providing a smooth solution for the entire flow region. The main feature of the expression proposed for the defect region is the introduction of an additional term in the wake function due to the blown or sucked fluid.

\section{THE EQUATIONS OF MEAN MOTION}

To study the equations of mean motion we follow Mellor's [1] procedure and assume that all components of the Reynolds stress tensor are of the same order; i.e. the axial and normal velocity fluctuations are of the same order. Now, considering that all lengths are non-dimensionalized by a typical body dimension, $l$, velocities by a characteristic velocity, $u_{\infty}$, kinematic pressure (pressure/density) by $u_{\infty}^{2}$, density by $\rho$ and viscosity by $\mu$, the non-dimensional governing equations of mean motion for steady twodimensional turbulent flow over flat surfaces can be written as

$$
\begin{gathered}
\frac{\partial U}{\partial X}+\frac{\partial V}{\partial Y}=0 \\
U \frac{\partial U}{\partial X}+(V+\tilde{\varepsilon} \tilde{V}) \frac{\partial U}{\partial Y}=-\frac{\partial P}{\partial X}+\frac{\partial}{\partial Y}\left(T_{x y}+\frac{1}{R} \frac{\partial U}{\partial Y}\right) \\
+\frac{\partial}{\partial X}\left(T_{x x}+\frac{1}{R} \frac{\partial U}{\partial X}\right)
\end{gathered}
$$

$$
\begin{aligned}
& U\left(\frac{\partial V}{\partial X}+\tilde{\varepsilon} \frac{\partial \tilde{V}}{\partial X}\right)+(V+\tilde{\varepsilon} \tilde{V}) \frac{\partial V}{\partial Y}=-\frac{\partial P}{\partial Y}+\frac{\partial}{\partial Y} \\
& \times\left(T_{y y}+\frac{1}{R} \frac{\partial V}{\partial Y}\right)+\frac{\partial}{\partial X}\left(T_{x y}+\frac{1}{R} \frac{\partial}{\partial X}(V+\tilde{\varepsilon} \tilde{V})\right)
\end{aligned}
$$

where $T_{x y}, T_{x x}$ and $T_{y y}$ are the dimensionless Reynolds stress components and the small parameters $\varepsilon, \tilde{\varepsilon}$ and $\hat{\varepsilon}$ are defined by

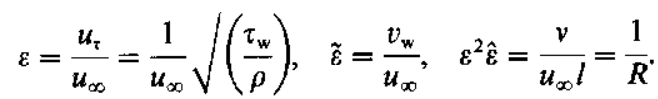

All the symbols in equations (1)-(3) have their classical meaning. The normal velocity, $V$, has been non-dimensionalized according to

$$
V=\frac{v-v^{*}}{u_{\infty}}
$$

Here lower case variables denote dimensional quantities. The normal velocity at the wall is given by $v^{*}=v_{\mathrm{w}} \tilde{V}(X)$, where $v_{\mathrm{w}}$ is a dimensional constant and $\tilde{V}(X)(=O(1))$ is a non-dimensional function. Due to transformation (5) the solution of the system of equations (1)-(3) is required to satisfy the no-slip and no-permeability conditions at the wall, that is

$$
U(X, 0)=V(X, 0)=0 .
$$

Upstream the flow has to agree with some previously prescribed velocity profile.

It is of interest to note that parameters $\varepsilon$ and $\hat{\varepsilon}$ define a singular perturbation problem whereas parameter $\tilde{\varepsilon}$ defines a regular perturbation problem. Indeed both small parameters $\varepsilon$ and $\hat{\varepsilon}$ multiply one of the highest 
derivative terms in the momentum equation. As this term is the laminar stress term one can easily see that as $\varepsilon, \hat{\varepsilon} \rightarrow 0$ the no-slip condition cannot be satisfied by the solution of the resulting system of equations. Thus small parameters $\varepsilon$ and $\hat{\varepsilon}$ characterize a singular perturbation problem and determine the length scales of the two inner regions, the defect layer and the wall layer. Hence, as mentioned in the introduction, the problem of turbulent boundary layers is characterized by three distinct scales, an inviscid scale, $l$, related to the external flow, an outer scale, $\delta(=\varepsilon l)$, related to the defect layer, and an inner scale, $\delta\left(=v / u_{\tau}=\hat{\varepsilon} \delta\right)$ related to the wall layer.

\section{ASYMPTOTIC ANALYSIS}

The main focus of this work is on the two inner layers. For this reason, the inviscid layer, despite being a necessary part of the total boundary layer problem, is not considered here. The task of obtaining the proper dependence of the asymptotic expansions on the two singular parameters is standard and hence is omitted here. Also, it is assumed that the asymptotic expansions can be expanded in integer powers of the regular parameter $\tilde{\varepsilon}$.

\subsection{Defect layer}

For this region we write

$$
\begin{aligned}
u=u_{11}(X, y)+ & \varepsilon u_{21}(X, y)+\tilde{\varepsilon} u_{12}(X, y) \\
& +\varepsilon \tilde{\varepsilon} u_{22}(X, y)+\varepsilon^{2} u_{31}(X, y)+\cdots \\
v=\varepsilon\left\{v_{11}(X, y)\right. & +\varepsilon v_{21}(X, y)+\tilde{\varepsilon} v_{12}(X, y) \\
& \left.+\varepsilon \tilde{\varepsilon} v_{22}(X, y)+\varepsilon^{2} v_{31}(X, y)+\cdots\right\} \\
p=p_{11}(X, y)+ & \varepsilon p_{21}(X, y)+\tilde{\varepsilon} p_{12}(X, y) \\
& +\varepsilon \tilde{\varepsilon} p_{22}(X, y)+\varepsilon^{2} p_{31}(X, y)+\cdots \\
\tau_{i j}=\varepsilon^{2} \tau_{i j_{31}}(X, y) & +\varepsilon \tilde{\varepsilon} \tau_{i j_{23}}(X, y) \\
& +\tilde{\varepsilon}^{2} \tau_{i j_{33}}(X, y)+\varepsilon^{3} \tau_{i j_{41}}(X, y)+\cdots
\end{aligned}
$$

where $y(=Y / \varepsilon)$ is the stretched normal coordinate for the defect layer.

Note that instead of assuming that the Reynolds stress tensor is given by expression ( $7 \mathrm{~d}$ ) we could alternatively have assumed that the fluctuations, $\boldsymbol{u}^{\prime}$ and $v^{\prime}$, have the form

$$
\begin{aligned}
u^{\prime} & =\varepsilon u_{21}^{\prime}(X, y)+\tilde{\varepsilon} u_{12}^{\prime}(X, y)+\cdots \\
v^{\prime} & =\varepsilon v_{21}^{\prime}(X, y)+\tilde{\varepsilon} v_{12}^{\prime}(X, y)+\cdots
\end{aligned}
$$

and hence expression (7d) would follow automatically.

The form of expansion ( $7 \mathrm{~d}$ ) can be arrived at using Prandtl's mixing-length theory together: th the assumption that the mixing length is proportional to the distance of the wall and expansion (7a). Also an analysis of the equations for the wall layer reveals the form of expansion ( $7 \mathrm{~d})$.

Substituting equations (7a)-(7d) into the equations of motion and grouping terms of the same order of magnitude, one can show that the first corrections to the undisturbed pressure profile are of order $\varepsilon^{2}$ and $\tilde{\varepsilon}^{2}$. It can also be shown that the Reynolds stress contributions appear in all equations of order higher than unity.

For a uniform oncoming stream condition, the lowest order equations together with the matching conditions for the inviscid and defect layers give

$$
u_{11}(x, y)=p_{11}(x, y)=1 \text {. }
$$

\subsection{Wall layer}

For this region we write

$$
\begin{aligned}
& \hat{u}=\varepsilon \hat{u}_{21}(X, \hat{y})+\tilde{\varepsilon} \hat{u}_{12}(X, \hat{y}) \\
&+\varepsilon^{2} \hat{u}_{31}(X, \hat{y})+\tilde{\varepsilon}^{2} \hat{u}_{13}(X, \hat{y})+\cdots \\
& \hat{v}=\varepsilon \hat{\varepsilon}\left\{\varepsilon \hat{v}_{21}(X, \hat{y})+\tilde{\varepsilon} \hat{v}_{12}(X, \hat{y})\right. \\
&\left.+\varepsilon^{2} \hat{v}_{31}(X, \hat{y})+\tilde{\varepsilon}^{2} \hat{v}_{13}(X, \hat{y})+\cdots\right\} \\
& \hat{p}=\hat{p}_{11}(X, \hat{y})+ \varepsilon \hat{p}_{21}(X, \hat{y}) \\
&+\tilde{\varepsilon} \hat{p}_{12}(X, \hat{y})+\varepsilon^{2} \hat{p}_{31}(X, \hat{y})+\cdots \\
& \hat{\tau}_{i j}=\varepsilon^{2} \hat{\tau}_{i j_{31}}(X, \hat{y})+\varepsilon \tilde{\varepsilon} \hat{\tau}_{i j_{22}}(X, \hat{y}) \\
& \quad+\tilde{\varepsilon}^{2} \hat{\tau}_{i j_{13}}(X, \hat{y})+\cdots
\end{aligned}
$$

where $\hat{y}(=Y / \varepsilon \hat{\varepsilon})$ is the stretched normal coordinate for the wall layer.

The several order equations for this layer are:

to $O(\varepsilon)$

$$
\begin{aligned}
\hat{u}_{21_{x}}+\hat{v}_{2 t_{y}} & =0 \\
\hat{u}_{21_{y p}}+\hat{\tau}_{x y_{3 t_{y}}} & =0 \\
\hat{p}_{21_{y}} & =0
\end{aligned}
$$

to $O(\tilde{E})$

$$
\begin{aligned}
\hat{u}_{12_{x}}+\hat{v}_{12_{\dot{y}}} & =0 \\
\hat{\tau}_{x y_{13_{y}}} & =\tilde{V} \hat{u}_{12_{\tilde{y}}} \\
\hat{p}_{12_{\hat{y}}} & =0 ;
\end{aligned}
$$

to $O(\varepsilon \tilde{\varepsilon})$

$$
\begin{aligned}
\hat{u}_{22_{x}}+\hat{v}_{22_{j}} & =0 \\
\hat{u}_{12_{y j}}+\hat{\tau}_{x y_{22_{\hat{y}}}} & =\hat{V} \hat{u}_{21_{y}} \\
\hat{p}_{22_{\hat{y}}} & =\hat{\tau}_{y y_{2 z_{\hat{y}}}} .
\end{aligned}
$$

In order to obtain a solution for equation (11b) we assume that as $\hat{y} \rightarrow \infty$ the laminar stress term becomes negligible. With this hypothesis and the Reynolds stress related to the mean velocity by standard mixinglength theory equations ( $11 \mathrm{~b}$ ) and (12b) can be solved. The mixing length is assumed to be proportional to the distance of the wall. For constant injection, $\tilde{V}=1$, the general solutions of equations (1Ib) and (12b) are 


$$
\begin{aligned}
& \hat{u}_{21}=\frac{1}{K} \ln \hat{y}+\hat{A} \\
& \hat{u}_{12}=\hat{B} \ln ^{2} \hat{y}+\hat{C} \ln \hat{y}+\hat{D}
\end{aligned}
$$

where $\hat{B}=1 / 4 K^{2}$ and $\hat{A}, \hat{C}$ and $\hat{D}$ are parameters to be determined.

An important feature of expression (14b) is that no relationship has been imposed between parameters $\hat{A}$, $\hat{C}$ and $\hat{D}$. Substitution of expressions (14a) and (14b) into expression (10a) gives the expression for the velocity profile in the wall layer, that is

$$
\begin{aligned}
\hat{u}(X, \hat{y})=\varepsilon\left(\frac{1}{K} \ln \hat{y}\right. & +\hat{A}) \\
& +\tilde{\varepsilon}\left(\frac{1}{4 K^{2}} \ln ^{2} \hat{y}+\hat{C} \ln \hat{y}+\hat{D}\right) .
\end{aligned}
$$

Expressions similar to equation (15) have been derived by several authorst who usually based their predictions on the same set of hypotheses. Here we review one of these approaches to illustrate the differences between our analysis and those carried out in previous works.

The usual approximation is to assume that there is a region near the wall where convection terms can be neglected and the shear stress is given by mixing length theory. Thus the equations of motion can be written as

$$
v_{\mathrm{w}} \frac{\mathrm{d} u}{\mathrm{~d} y}=\frac{\mathrm{d} \tau}{\mathrm{d} y}=\frac{\mathrm{d}}{\mathrm{d} y}\left[K^{2} y^{2}\left(\frac{\mathrm{d} u}{\mathrm{~d} y}\right)^{2}\right]
$$

This equation integrated twice with the condition $\tau=\tau_{\mathrm{w}}$ at $y=0$, gives

$$
\frac{1}{K} \ln y+d=\frac{2}{v_{\mathrm{w}}}\left(v_{\mathrm{w}} u+u_{\mathrm{\tau}}^{2}\right)^{1 / 2}
$$

where $d$ is a parameter of integration.

The difficulty with equation (17) is that it does not reduce to the no-blowing law of the wall as $v_{\mathrm{w}} \rightarrow 0$. This difficulty was rectified by Stevenson [5] in the following way. First he re-wrote equation (17) as

$$
\frac{1}{K} \ln \hat{y}+\hat{d}=\frac{2 u_{\tau}}{v_{w}}\left(v_{w} \frac{u}{u_{\tau}^{2}}+1\right)^{1 / 2} .
$$

Next he added the term $2 u_{\mathrm{t}} / v_{\mathrm{w}}$ to both sides of equation (18) to obtain

$\frac{2 u_{\tau}}{v_{w}}\left\{\left(\frac{v_{w} u}{u_{\tau}^{2}}+1\right)^{1 / 2}-1\right\}=\frac{1}{K} \ln \hat{y}+\left(\hat{d}-\frac{2 u_{\tau}}{v_{w}}\right)$.

Then reasoning that the term $\left(\hat{d}-2 u_{\imath} / v_{w}\right)$ varies very little with injection or suction he obtained an expression which can be written as

$\dagger$ For details see, e.g. ref. [5].

$$
u=\varepsilon\left(\frac{1}{K} \ln \hat{y}+A\right)+\tilde{\varepsilon}\left(\frac{1}{4 K^{2}} \ln ^{2} \hat{y}+\frac{A}{2 K} \ln \hat{y}+\frac{A^{2}}{4}\right)
$$

where $A=\left(\hat{d}-2 u_{\tau} / v_{\mathrm{w}}\right)$ is the constant for the unblown case.

Expression (20) is the classical law of the wall for transpired turbulent boundary layers. Note that expressions (15) and (20) have the same general form. However, a basic difference between these two equations is that equation (15) results from the solutions of two distinct equations whereas equation (20) results from the solution of equation (16) only. Thus one of the consequences of the asymptotic analysis is that it does not impose a priori any relationship between the parameters multiplying the logarithmic, bilogarithmic and independent terms in the law of the wall. Therefore, any such relationship should be determined experimentally. The previous theories on the other hand tie up all the coefficients in the law of the wall. Furthermore, they normally assume that these parameters are independent of the injection or suction rate and hence can be obtained from the unblown case. This latter hypothesis does not seem to hold since the results of recent research have suggested that parameter $A$ decreases with increasing injection rate. In any event expressions with the form of equations (15) and (20) normally give a fair agreement with the experimental data.

Having obtained an expression for the solution in the wall layer, we now shift our attention to the defect layer. To have an indication of the form of the velocity profle in the defect layer we re-write expression (15) in outer variables and obtain

$$
\begin{aligned}
\hat{u}= & \varepsilon\left(\frac{1}{K} \ln y+\frac{1}{K} \ln \frac{\delta}{\delta}+\hat{A}\right)+\tilde{\varepsilon}\left[\frac{1}{4 K^{2}} \ln ^{2} y\right. \\
& \left.+\left(\frac{1}{2 K^{2}} \ln \frac{\delta}{\delta}+\hat{C}\right) \ln y+\frac{1}{4 K^{2}} \ln ^{2} \frac{\delta}{\delta}+\hat{C} \ln \frac{\delta}{\delta}+\hat{D}\right] .
\end{aligned}
$$

Now, if there is to be an overlap region between the inner and outer layers, the defect expression as $y \rightarrow 0$ has to agree with equation (21) at any point $y$ in this domain. This can be accomplished by an expression with the form shown below

$$
\begin{aligned}
u=1 & +\varepsilon\left(\frac{1}{K} \ln y-\frac{\pi}{K}[2-w(y)]\right) \\
& +\widetilde{\varepsilon}\left(B \ln ^{2} y+C \ln y-\frac{\tilde{\pi}}{K}[2-w(y)]\right)+\cdots
\end{aligned}
$$

where $\pi$ is Cole's profile parameter, $w(y)$ is Cole's wake function, $\tilde{\pi}$ the profile parameter of the wake component due to the transpiration, and $B, C$ the parameters to be determined.

If only matching arguments are considered, $B$ and 
$C$ can in general be any function of $y$ having the form illustrated by function $F$

$$
F(y)=\text { const } F_{1}(y)+F_{2}(y)
$$

where

$$
\begin{array}{ll}
\lim F_{1}(y)=1, & \text { as } y \rightarrow 0 \\
\lim F_{2}(y)=0, & \text { as } y \rightarrow 0 .
\end{array}
$$

The conditions to be satisfied at the edge of the turbulent boundary layer are

$$
\begin{aligned}
\lim B(y) \ln ^{2} y=0, & \text { as } y \rightarrow 1 \\
\lim C(y) \ln y=0, & \text { as } y \rightarrow 1 .
\end{aligned}
$$

Of course this opens a wide range of possibilities to be considered. The simplest of these possibilities is to take $D$ and $C$ as being independent of $y$. Then the matching principle applied to equations (21) and (22) requires that

$$
\begin{gathered}
B=\hat{B}=\frac{1}{4 K^{2}} \\
C=\frac{1}{2 K^{2}} \ln \frac{\delta}{\delta}+\hat{C}
\end{gathered}
$$

and

$$
\begin{aligned}
1=\varepsilon\left(\frac{1}{K} \ln \frac{\delta}{\delta}+\hat{A}+\frac{2 \pi}{K}\right) \\
\quad+\tilde{\varepsilon}\left(\frac{1}{4 K^{2}} \ln ^{2} \frac{\delta}{\delta}+\hat{C} \ln \frac{\delta}{\delta}+\hat{D}+\frac{2 \tilde{\pi}}{K}\right) .
\end{aligned}
$$

Equation (23c) is an extension of the well-known turbulent skin-friction equation [1] to the case with transpiration. Its complete definition depends now on the determination of parameters $\hat{A}, \hat{C}, \hat{D}$ and $\tilde{\pi} . \tilde{\pi}$ is a parameter which is considered independent of $x$ and $y$.

The main feature of equation (22) is the introduction of a universal function in the transpired component of the mean velocity profile with a profile parameter, $\pi$, distinct from $\pi$. This function is here assumed to be identical to Cole's wake function.

One of the features of the analysis carried out so far is that no proper boundary conditions are available for evaluating the constants in the proposed expressions. Consequently these parameters have to be determined through conditions supplemented by experimental works.

\section{THE LAW OF THE WAKE FOR INCOMPRESSIBLE TTBL}

Assuming that Stevenson's law of the wall formulation provides good theoretical predictions, it follows from equations (15) and (20) that

$$
\hat{A}=A
$$

$$
\hat{C}=A / 2 K
$$

and

$$
\hat{D}=A^{2} / 4
$$

As a logical extension to the law of the wall Stevenson [6] proposed the following expression for the defect layer :

$$
\begin{aligned}
2 \frac{u_{\tau}}{v_{w}}\left[\left(1+\frac{v_{\mathrm{w}} u_{\infty}}{u_{t}^{2}}\right)^{1 / 2}\right. & \left.-\left(1+\frac{v_{w} u}{u_{\tau}^{2}}\right)^{1 / 2}\right] \\
& =-\frac{1}{K} \ln y+\psi(1)-\psi(y)
\end{aligned}
$$

where $\psi$ is a universal function.

This expression has been tested by the above author showing good agreement with his experimental results. To allow a direct comparison between expressions (22) and (27) we re-write expression (27) as

$$
\begin{aligned}
u=1+\varepsilon \frac{1}{K}(1+X)^{1 / 2} \ln y-\varepsilon \psi^{\prime}(1+X)^{1 / 2} & \\
& +\tilde{\varepsilon} \frac{1}{4 K^{2}} \ln ^{2} y-\tilde{\varepsilon} \frac{1}{2 K} \psi^{\prime} \ln y+\frac{\tilde{\varepsilon}}{4} \psi^{\prime 2}
\end{aligned}
$$

where

$$
X=v_{w} u_{\infty} / u_{\tau}^{2}
$$

and

$$
\psi^{\prime}=\psi(1)-\psi(y)
$$

From ref. [6] it can be shown that

$$
(1+X)^{1 / 2}=1+\frac{v_{w}}{2 u_{\tau}} \frac{A}{K} \ln \frac{\delta u_{\tau}}{v}+\frac{v_{\mathrm{w}}}{2 u_{\tau}} \psi(1) .
$$

The coefficients of the bilogarithmic terms in expression (22) and in equation (28) agree exactly. Substitution of equation (31) into equation (28) shows that the coefficients of the logarithmic terms also agree since

$$
\begin{aligned}
\frac{\varepsilon}{K}+\tilde{\varepsilon} \frac{1}{2 K^{2}} \ln \frac{\delta u_{\tau}}{v} & +\tilde{\varepsilon} \frac{A}{2 K}=-\frac{\tilde{\varepsilon}}{2 K} \psi(1)+\tilde{\varepsilon} \frac{1}{2 K} \psi(0) \\
& +\frac{\varepsilon}{K}+\tilde{\varepsilon} \frac{1}{2 K^{2}} \ln \frac{\delta u_{\tau}}{v}+\frac{\tilde{\varepsilon}}{2 K} \psi(1) .
\end{aligned}
$$

A comparison between the independent terms shows that here we have replaced the term

$$
\frac{1}{4}[\psi(1)-\psi(y)]^{2}-\frac{A}{2}[\psi(1)-\psi(y)] \ln \frac{\delta u_{\tau}}{v}+\frac{\psi}{2}(1)
$$

in Stevenson's expression by the simpler term $\tilde{\pi}[2-w(y)] / K$ where $\tilde{\pi}$ is a function of $v_{w}$.

Our expression for the defect layer for incompressible transpired turbulent boundary layers then reads 


$$
\begin{aligned}
u=1 & +\varepsilon\left(\frac{1}{K} \ln y-\frac{\pi}{K}[2-w(y)]\right)+\tilde{\varepsilon}\left[\frac{1}{4 K^{2}} \ln ^{2} y\right. \\
& \left.+\left(\frac{1}{2 K^{2}} \ln \frac{\delta}{\delta}+\frac{A}{2 K}\right) \ln y-\frac{\tilde{\pi}}{K}[2-w(y)]\right] .
\end{aligned}
$$

\section{THE DETERMINATION OF A AND $\tilde{\pi}$}

Various investigators have presented results which suggest that $A$ varies with transpiration. Indeed, it is now a well-accepted fact that strong injection rates reduce the value of $A$. Thus the idea of a non-constant $A$ is plausible and will be investigated in this section.

From a full consideration of the accuracy and repeatability of 200 measured boundary layer developments with transpiration, Squire [8] recommends as basic test cases the flows with zero pressure gradient and constant injection as measured by Andersen [9]. These experimental results will then be used to determine parameters $A$ and $\tilde{\pi}$.

The experimental flow conditions to be considered are summarized in Table 1.

Since no conclusion has been reached yet as to which parameters should be used to account for the variation of $A$ and if so, in which way these parameters should be non-dimensionalized, we assumed here for gain of simplicity that $A$ is a function of $\tilde{\varepsilon}$ only. Therefore, any dependence of $A$ on the momentum thickness, $\theta$, and on $C_{\mathrm{f}}$, has been neglected throughout this work.

In order to study the dependence of $A$ on the value of the injection rate, Andersen's experimental points were plotted in the form $\phi$ vs $\log \hat{y}$, where $\hat{y}=Y u_{\tau} / v$, $u^{+}=U / u_{\tau}, v^{+}=V_{\mathrm{w}} / u_{\tau}$ and $\phi$ has been defined in Fig. 1. Hence if the obtained profiles are fitted by straight lines, the slopes determine the values of $K$ whereas the level of the curves determine the values of $A$. The results are shown in Fig. 1 and Table 2.

So, from Fig. 1, it can be seen that (1) $K$ does not change with different injection rates and its value seems to be 0.41 , (2) $A$ does change with distinct injection rates varying from 4.80 to 0.65 .

The experimental results plotted as $A$ vs $\tilde{\varepsilon}$ and fitted by a straight line determine, as shown in Fig. 3, the following expression :

$$
A=5.0-512 \tilde{\varepsilon} \text {. }
$$

Expression (35) has to be regarded with some caution because $A$ depends critically on the measured values of $C_{\mathrm{f}}$ and therefore a small error in $C_{\mathrm{f}}$ would

Table 1. Experimental flow conditions

\begin{tabular}{llc}
\hline$F I$ & \multicolumn{1}{c}{$\tilde{\varepsilon}$} & $U_{\infty}\left[\mathrm{ft} \mathrm{s}^{-1}\right]$ \\
\hline 1 & 0.0 & 31.0 \\
2 & 0.00102 & 31.0 \\
3 & 0.00200 & 31.0 \\
4 & 0.00375 & 31.0 \\
5 & 0.00800 & 31.0 \\
\hline
\end{tabular}

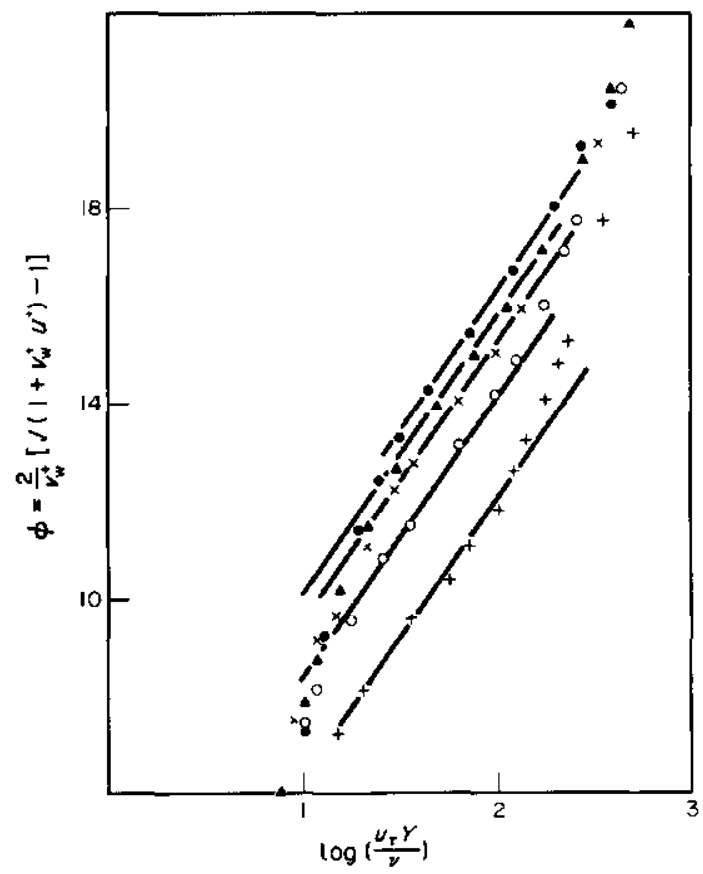

FiG. 1. Variation of $\phi$ with $Y u_{\tau} / v$ according to Andersen : , $F=1 ; \boldsymbol{\Delta}, F=2 ; \times, F=3 ; O, F=4 ;+, F=5$.

Table 2. Values of $A$ according to Andersen

\begin{tabular}{lcc}
\hline \multicolumn{1}{c}{$\tilde{\varepsilon}$} & $K$ & $A$ \\
\hline 0.0 & 0.41 & 4.80 \\
0.00102 & 0.41 & 4.34 \\
0.00200 & 0.41 & 3.80 \\
0.00375 & 0.41 & 2.65 \\
0.00800 & 0.41 & 0.65 \\
\hline
\end{tabular}

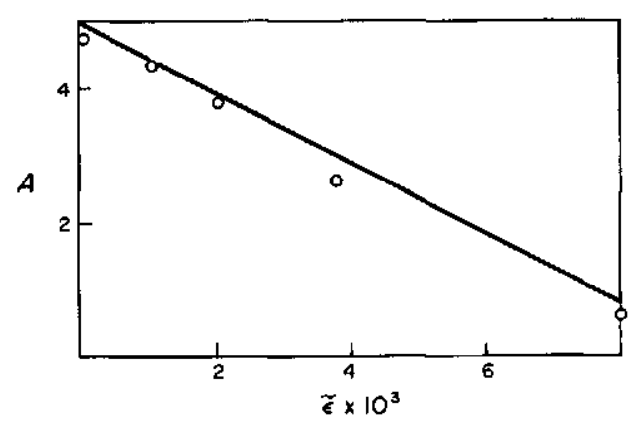

Fig. 2. Variation of $A$ with $\tilde{\varepsilon}$.

produce different values of $A$ and consequently a different expression for its variation. It is however very difficult to propose a universal and definitive expression for $A$ due to the general lack of agreement between the various sets of data available in the literature. Thus it should be noted that most of the investigators obtained their skin-friction values from some form of momentum equation which tends to be inaccurate, especially for high injection rates. Indeed, Dahm and Kendall [10] point out that values of $C_{\mathrm{r}}$ obtained from the two-dimensional momentum inte- 


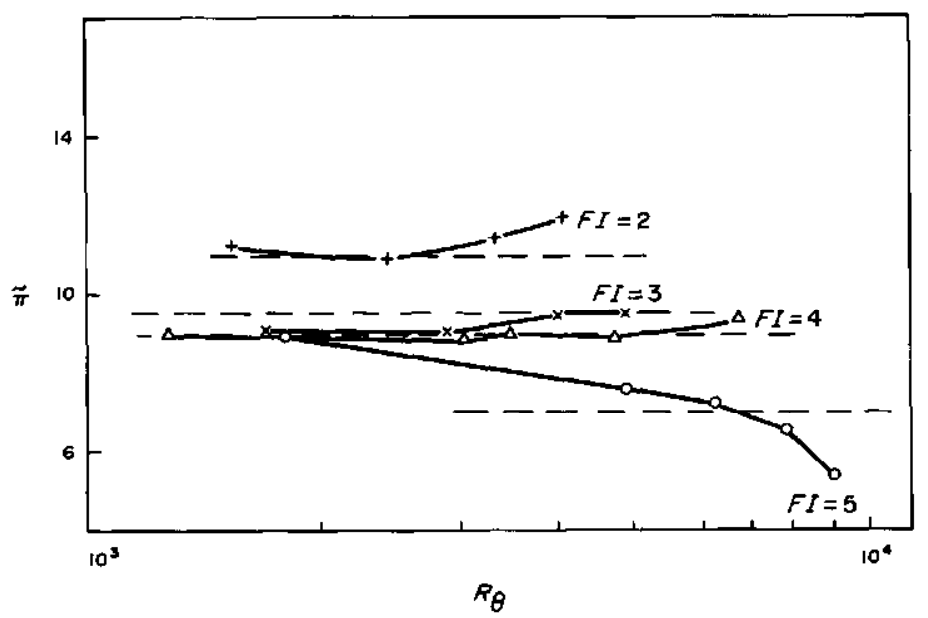

FIG. 3. Variation of $\tilde{\pi}$ with $\tilde{\varepsilon}$ and $R_{\theta}$.

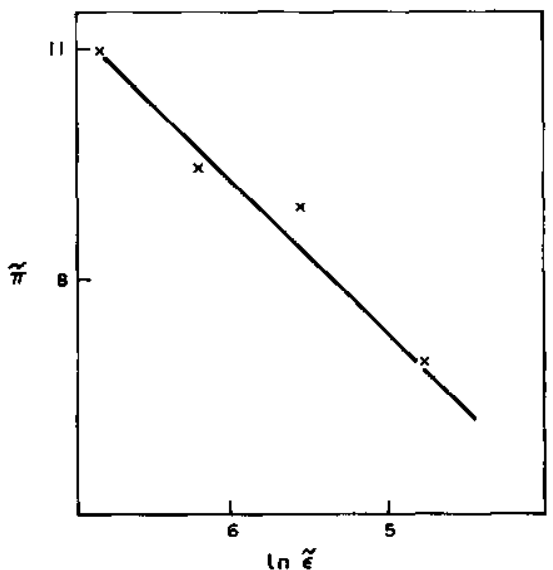

FIG. 4. Mean values of $\tilde{\pi}$ against $\tilde{\varepsilon}$.

gral equation depend strongly both on the accuracy of the knowledge of the momentum thickness variation with the fluid flow distance from the leading edge $(\mathrm{d} \theta / \mathrm{d} x)$ and on the accuracy of the blowing rate $\left(F=\rho_{\mathrm{w}} V_{\mathrm{w}} / \rho_{\infty} U_{\infty}\right)$. They show that at a blowing rate of 0.005 at $R_{x}=10^{6}$, an uncertainty of $\pm 1 \%$ in both $\mathrm{d} \theta / \mathrm{d} x$ and $V_{\mathrm{w}} / U_{\infty}$ yields an uncertainty of $\pm 32 \%$ in $C_{r} / 2$. In view of these remarks and the fact that Andersen made redundant measurements to check his results we assume in this work that expression (35) describes reasonably well the variation of $A$.

Parameter $\tilde{\pi}$ will be determined using equation (23c) and the experimental data of Andersen.

From equation (23c) it follows:

$$
\begin{aligned}
1= & \varepsilon\left(\frac{1}{K} \ln \frac{\delta u_{\tau}}{v}+A+\frac{2 \pi}{K}\right) \\
& +\tilde{\varepsilon}\left(\frac{1}{4 K^{2}} \ln ^{2} \frac{\delta u_{\tau}}{v}+\frac{A}{2 K} \ln \frac{\delta u_{\tau}}{v}+\frac{A^{2}}{4}+\frac{2 \tilde{\pi}}{K}\right) .
\end{aligned}
$$

With values of $\delta, u_{t}, V_{\mathrm{w}}$ and $U_{\infty}$ obtained from the work of Andersen, equation (36) can be solved to determine $\tilde{\pi}$. Values of $\tilde{\pi}$ obtained through this process are presented in Fig. 3 for several injection rates and values of $\boldsymbol{R}_{\boldsymbol{\theta}}$. The results of Andersen suggest that $\tilde{\pi}$ is invariant with respect to $R_{\theta}$ and tends to decrease with increasing injection.

One of the inadequacies of the experimental results in Fig. 3 is that they cover a very low $\boldsymbol{R}_{\theta}$.range and under these circumstances one cannot be completely sure of the value of $\tilde{\pi}$ in equilibrium conditions. In this work we follow the trend suggested by Andersen's data and assume that $\bar{\pi}$ is independent of $R_{\theta}$ and decreases with increasing injection rate. The curves for the same injection rate are then approximated by the intermittent lines as shown in Fig. 3. These mean values plotted against $\tilde{\varepsilon}$ and fitted by a straight line determine, as shown in Fig. 4, the following expression:

$$
\tilde{\pi}=-1.95 \ln \tilde{\varepsilon}-3.1
$$

Predictions of $C_{f}$ using equations (36) and (37) are shown in Fig. 5. As expected, the agreement for weak and moderate injection rates is very good. Typical velocity profiles obtained using equation (34) are shown in Fig. 6. Again the agreement here is very good. It is important to note that the velocity profiles have been obtained with values of $\tilde{\pi}$ determined to satisfy the skin-friction equation and that for this reason there was a priori no guarantee that the defect layer solution would also give good predictions.

\section{ANALYSIS OF THE SUCTION RESULTS}

Several investigators have studied the problem of incompressible turbulent boundary layers with uniform suction. These experiments are particularly valuable for testing theories for transpired turbulent boundary layers since, in contrast to the blowing case, skin-friction coefficients can be determined very accurately using the two-dimensional momentum equation. In order to test equation (23c) for the exper- 


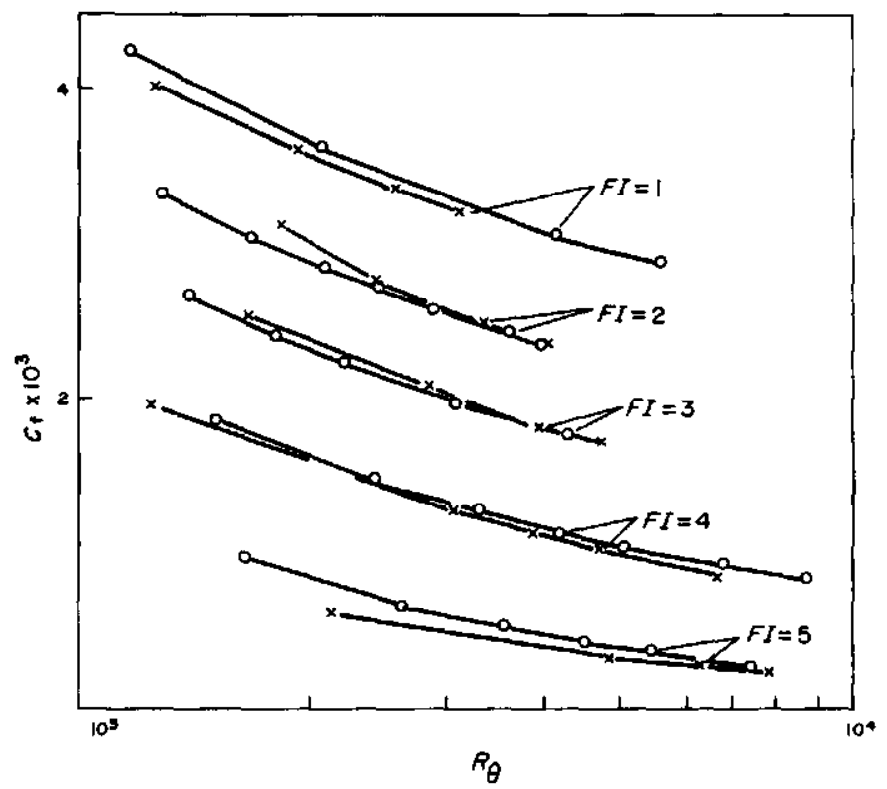

Fug. 5. Comparison of results for various injection rates and $R_{\theta}: O$, equation (36); $\times$, Andersen's results.

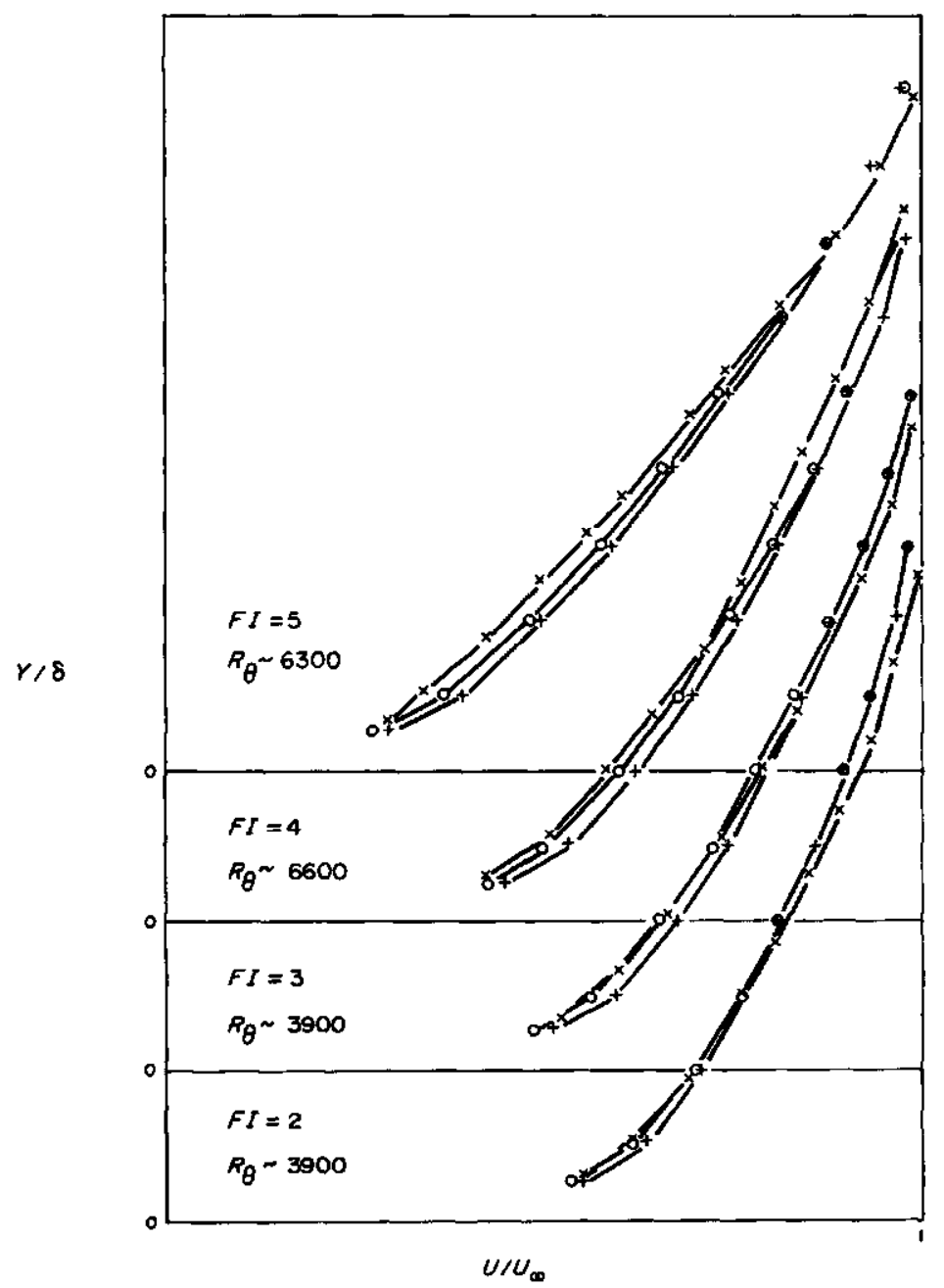

FIG. 6. Velocity profile correlation: $\times$, experimental data; $O$, equation (34); +, equation (28). 


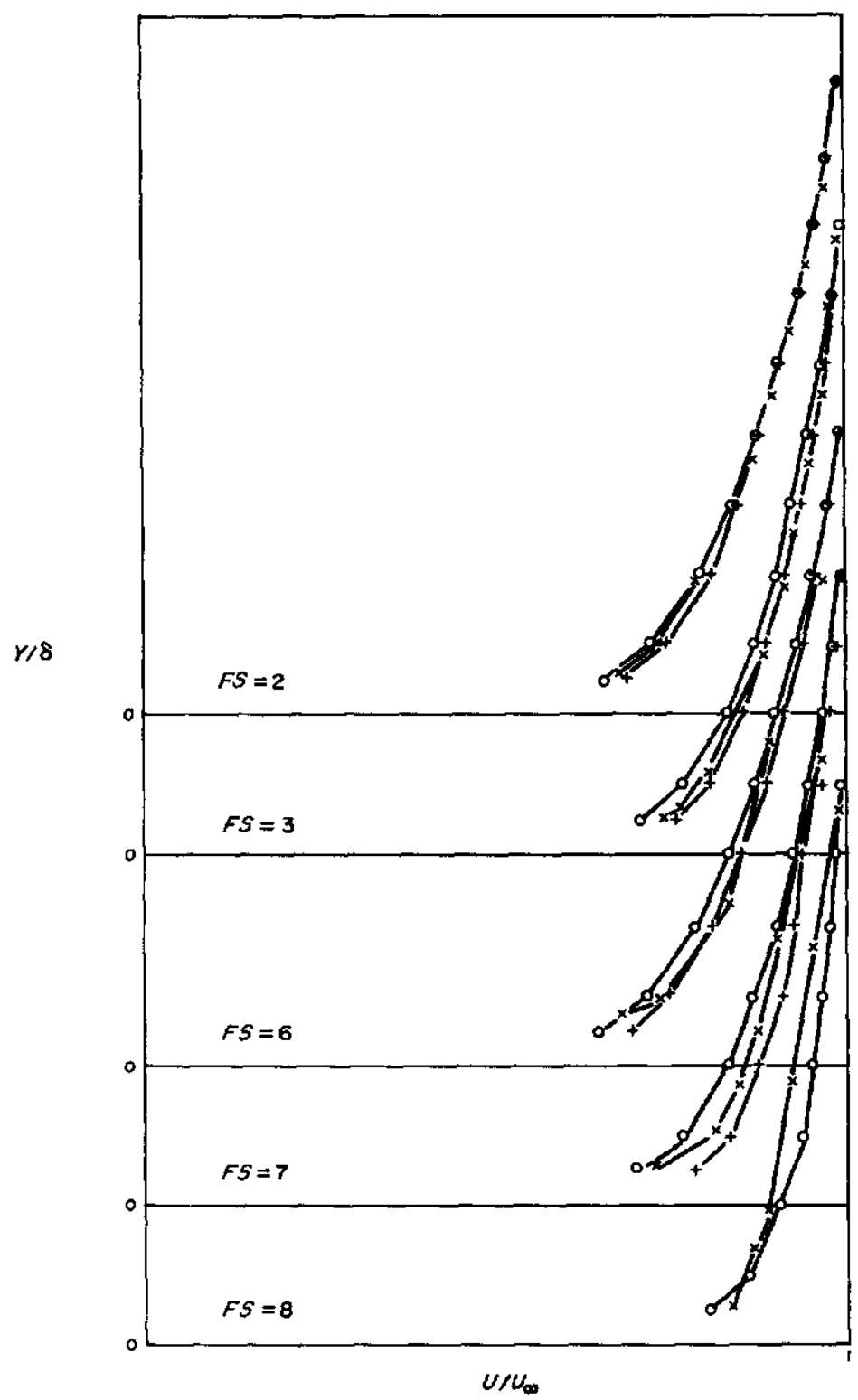

FIG. 7. Velocity profile correlation: $x$, experimental data; $O$, equation (34); + , equation (28).

imental data for sucked flow, we have chosen the works of Simpson et al. [11] and Favre et al. [12] since both groups studied zero-pressure gradient flow for several different suction rates. A basic difference between these two works, however, lies on the fact that Simpson et al. applied suction from the leading edge of the test surface whereas Favre $e t$ al. applied suction after a long stretch of solid surface. Thus the layer obtained by Simpson $e t$ al. for high suction rates was very thin closely approximating the asymptotic state. The experimental conditions considered here and the values of $\varepsilon$ predicted by equation (23c) are shown in Table 3 . Since now the normal velocity at the wall has a negative value we have used the absolute value of $\tilde{\varepsilon}$ in equation (37).

As can be seen, the theoretical predictions are very good. Note that the results for sucked flow have been obtained using the expression derived for blown flow and the numerical values of the constants have not

Table 3. Suction results

\begin{tabular}{lcccccr}
\hline Investigators & $F S$ & $\tilde{\varepsilon}$ & Theor. & Exp. & $R_{\delta}$ \\
\hline Simpson et $\alpha l$. & 1 & -0.00115 & 0.0474 & 0.0469 & 31010 \\
& 2 & -0.00117 & 0.0483 & 0.0482 & 24070 \\
& 3 & -0.00231 & 0.0554 & 0.0532 & 22620 \\
& 4 & -0.00242 & 0.0576 & 0.0558 & 12840 \\
Favre et al. & 5 & -0.00462 & 0.0770 & 0.0663 & 1526 \\
& 6 & -0.00119 & 0.0493 & 0.0469 & 18570 \\
& 7 & -0.00252 & 0.0573 & 0.0534 & 17730 \\
& 8 & -0.00516 & 0.0730 & 0.0663 & 15960
\end{tabular}


been adjusted in any way. The discrepancy found for the predicted value of $\varepsilon$ for Simpson et al.'s highest suction data are to be expected, since in these conditions the turbulent fluctuations in the boundary layer are completely removed, resulting in a laminar layer in an asymptotic state. In these conditions the assumptions which led to the derivation of equation (23c) do not hold.

The theoretical and experimental velocity profiles obtained are presented in Fig. 7. Again the agreement is very good. The results obtained by Stevenson's defect expression (equation (28)) have not been presented for Favre et al.'s highest suction rate because for this condition $B$ assumes the value -1.174 and therefore the operation $(1+B)^{1 / 2}$ cannot be carried out. Thus Stevenson's expression does not provide any predictions for $\tilde{\varepsilon}=-0.00516$.

\section{FINAL REMARKS}

Perturbation techniques have been used in this work in order to obtain solutions for the two inner regions associated with the boundary layer. We have shown how these equations lead to a bilogarithmic expression for the law of the wall and how this law can be extended to the defect layer. The present formulation also determines an equation for the skinfriction coefficient which has been successfully tested against experimental data. Another attractive feature of this analysis is that the incompressible results can be extended for compressible flow by means of a Van Driest transformation; thus providing a defect law and a skin-friction equation for transpired compressible turbulent boundary layers. An investigation of the results of such a procedure has been carried out by this author for several Mach numbers and injection rates and will be published shortly.
Acknowledgements-The author wishes to acknowledge, with gratitude, Dr L. C. Squire for his help and advice during the preparation of this research work. Useful comments were also received from Dr A. P. Dowling and Mr N. Chokani. This work was financially supported partly by the Brazilian National Research Council (Conselho Nacional de Desenvolvimento Cientifico e Tecnologico) and partly by an ORS award.

\section{REFERENCES}

1. G. Mellor, The large Reynolds number asymptotic theory of turbulent boundary layers, Int. J. Fngng S Si. 10, 851-873 (1972).

2. K. S. Yajnik, Asymptotic theory of turbulent shear flows, J. Fluid Mech. 42, 4I 1-427 (1970).

3. N. Afzal, Effects of longitudinal curvature, vorticity and displacement for two-dimensional turbulent boundary layers with heat and mass transfer, J. Mec. 14, 824-841 (1975).

4. R. L. Simpson, Characteristics of turbulent boundary layers at low Reynolds number with and without transpiration, J. Fluid Mech. 42, 769-802 (1970).

5. T. N. Stevenson, A law of the wall for turbulent boundary layers with suction or injection, Cranfield College of Aero Rep Aero No. 166 (1963).

6. T. N. Stevenson, A modified velocity defect law for turbulent boundary layers with injection, Cranfield College of Aero Rep Aero No. 170 (1963).

7. J. McQuaid, Ph.D. thesis, Cambridge University (1966).

8. L. C. Squire, Turbulent boundary layers with suction or injection, Conference on Data and Computation for Complex Turbulent Flows Report of Evaluator (1980).

9. P. S. Andersen, W. M. Kays and R. J. Moffat, The turbulent boundary layer on a porous plate: an experimental study of the fluid mechanics for adverse freestream pressure gradients, Stanford University Report No. HMT-15 (1972).

10. T. J. Dahm and R. M. Kendall, Comments on "Inner region of transpired turbulent boundary layers", $A I A A$ J. 6, 1822-1824 (1968).

11. R. L. Simpson, W. M. Kays and R. J. Moffat, The turbulent boundary layer on a porous surface : an experimental study of the fluid dynamics with fluid injection or suction, Stanford University Report No. HMT-2 (1967).

12. A. Favre, R. Dumas, E. Verollet and M. Coantic, Couche limite turbulent sur paroi poreusse avec transpiration, J. Mec. 5, 3-28 (1966).

\title{
SOLUTION ASYMPTOTIQUE POUR DES COUCHES LIMITES TURBULENTES INCOMPRESSIBLES DE TRANSPIRATION
}

\begin{abstract}
Résumé-On utilise des techniques de perturbation pour étudier les équations du mouvement moyen pour des couches limites turbulentes transpirées. Une des originalités de cette approche est l'utilisation d'échelles multiples. La solution nécessite trois échelles distinctes associées à trois régions d'écoulement: la région non visqueuse, la couche intermédiaire et la couche pariétale. Dans la construction des solutions de paroi et intermédiaire, une nouvelle loi du sillage est déterminée par comparaison de ces deux solutions dans un intervalle du domaine commun de validité. On obtient une équation de frottement pariétal pour les couches limites turbulentes de transpiration. Les profils de vitesse obtenus à partir de la loi de sillage, et les valeurs du coefficient de frottement dans le cas des écoulements avec injection et succion sont comparés favorablement aux données expérimentales.
\end{abstract}




\title{
ASYMPTOTISCHE LOSUNG FUR INKOMPRESSIBLE TURBULENTE STRÖMUNGS- GRENZSCHICHTEN
}

Zusammenfassung-In der vorliegenden Arbeit wurde ein Verfahren der Störungsansätze dazu benutzt, die Gleichungen der mittleren Bewegung in inkompressiblen turbulenten Strömungs-Grenzschichten zu untersuchen. Eines der Merkmale dieser Näherungsmethode ist der Einsatz von unterschiedlichen MaBstabsfaktoren, nämlich jeweils einer in den drei Strömungs-Bereichen : nicht-viskoser Kernbereich, gestörte Schicht und Wandschicht. Der Aufbau der Lösung für die Wand- und die gestörte Schicht führte durch Vergleich dieser beiden Lösungen in einem Abschnitt von beiderseitiger Gültigkeit zu einer neuen Gesetzmäßigkeit für die Wirbelströmung. Die Schließ-Bedingungen ergeben auch eine Gleichung für die Oberflächenreibung in turbulenten Strömungs-Grenzschichten. Die Geschwindigkeitsprofile, die man aus der Gesetzmäßigkeit für die Wirbelströmung erhält, und die Werte des Oberflächenreibungskoeffizienten, die mit der Gleichung für die Oberflächenreibung der Strömungen in Flüssigkeiten mit Einspritzung und Absaugung ermittelt wurden wurden bei guter Übereinstimmung mit experimentellen Daten verglichen.

\section{АСИМПТОТИЧЕСКОЕ РЕПЕНИЕ ДЛЯ НЕСЖИМАЕМЫХ ТУРБУЛЕНТНЫХ ПОГРАНИЧНЫХ СЛОЕВ НА ПРОНИЦАЕМОЙ ПОВЕРХНОСТИ}

\begin{abstract}
Анғотаип-Методом возмущений исследованы уравнения среднего двихения для несжимаемых турбулентных пограничных слоев на проницаемой поверхности. Одной из особенностей метода является многомасштабность. В частности, для решения данной задачи использовались три масштаба для трех характерных областей течения: невязкого течения, слоя с дефектом скорости и пристенного слоя. При построении решений для пристенной зоны и области дефекта скорости найден новый закон следа путем сравнения решений в промехуточной области взаимодействня. Условия срашивания решений тахже удовлетворяют и уравнению поверхностного трения для рассматриваемого пограничного слоя с отсосом. Проведено сравнение с экспериментальными данными профилей скоросги, расчитанных с учетом полученного закона для следа, и коэффищента поверхностного трения, рассчитанного из уравнения поверхностного трения для пограничного слоя со вдувом и отсосом. Получено хорошее совпадение расчетных и зхспернментальных результатов.
\end{abstract}

